\title{
Stroke and pregnancy
}

\author{
C A Davie, P O’Brien
}

Department of Clinical

Neurosciences, The Royal Free Hampstead NHS Trust, Pond

Street, London, UK; Department of Obstetrics and Gynaecology,

University College London

Hospitals, London, UK

\section{Correspondence to:}

Dr C Davie, Consultant

Neurologist, Department of

Clinical Neurosciences. The

Royal Free Hampstead NHS

Trust, Pond Street, London NW3

20G, UK; c.davie@medsch.ucl. ac.uk

Received 26 July 2007

Revised 24 October 2007

Accepted 25 October 2007

Published Online First

6 November 2007

\section{ABSTRACT}

Pregnancy-related stroke is, fortunately, a rare event. However, when it occurs, there may be implications for management of the patient and delivery of the child. This article reviews the mechanisms and risk factors for stroke related to pregnancy, the presenting features, diagnosis and management of the different stroke types, and the implications for pregnancy and delivery.

Pregnancy (and the puerperium) are pro-thrombotic states. During pregnancy and for approximately 6 weeks after birth, all women are at increased risk of thromboembolic disease (TED). Indeed, in the last report on the Confidential Enquiries into Maternal Deaths in the UK, thromboembolism was the leading direct cause of maternal death. ${ }^{1}$

The reported incidence of pregnancy-related stroke varies widely, but probably lies between 11 and 26 deliveries per 100000 (table 1) ) $^{2-8}$. Studies showing an incidence at the lower end of this range may not be representative, given that a study in non-pregnant women of reproductive age reported an incidence of stroke at 10.7 per $100000{ }^{9}$ However, given the data available, it could be predicted that an average maternity unit in the UK delivering approximately 3300 births would expect to see a pregnancy-related stroke every 9 months to 2 years. For this reason, care of such women should be centralised in units with a multidisciplinary team (including a neurologist, obstetrician and rehabilitation services) that is experienced in dealing with such conditions. One study ${ }^{2}$ has shown a rate of ischaemic stroke of 11/100 000 deliveries with the postpartum period representing the greatest period of risk. In the same study, intracerebral haemorrhage occurred in 9/100 000 deliveries, again with the highest risk occurring in the postpartum period. This large hospital-based registry study concluded that the risk of ischaemic or haemorrhagic stroke was increased in the 6 weeks after pregnancy, but not during pregnancy itself. However, at least one retrospective series ${ }^{6}$ showed a tendency for arterial strokes to occur throughout pregnancy, with a higher incidence in the third trimester and postpartum period. The same study showed that most venous events occurred in the puerperium. A large Swedish cohort of more than 650000 women with more than 1 million deliveries in an 8-year time period concluded that the greatest risk of ischaemic and haemorrhagic cerebral events was in the 2 days before and 1 day after delivery, with an increased but declining risk over the subsequent 6-week postpartum period. ${ }^{8}$ It has been estimated that there is a mortality of $10-13 \%$ following pregnancy-related stroke and that this is disproportionately higher in black women, in older patients and in those with no prenatal care., ${ }^{5,10}$

\section{DIAGNOSIS}

As in other patients, it is important when a stroke does occur to establish as quickly as possible if this is due to cerebral ischaemia or haemorrhage. The clinical history or physical examination is insufficiently sensitive to answer this question and so brain imaging in the form of CT or MRI should be carried out as quickly as possible after symptom onset. Although animal studies have raised concerns about potential teratogenic effects of MRI exposure in early pregnancy, this remains the preferred imaging option in pregnancy. CT scanning does expose the foetus to radiation; however, if MRI is not available, the benefits of CT scanning greatly outweigh the risks in this situation and should be performed. As is the case with all youngonset stroke patients, there should be an extensive diagnostic assessment, including (when appropriate) vascular studies of the extracranial vessels, cardiac investigation and thrombophilia screening.

It is worth pointing out that migraine with an accompanying aura is still sufficiently common in pregnancy that it should be considered in the differential diagnosis, particularly if there is an evolving neurological deficit rather than an abrupt onset.

\section{ISCHAEMIC STROKE}

The risk of ischaemic events in pregnancy and the puerperium is influenced by ethnic background and by age. African American women have a significantly higher risk than Caucasians (there is little epidemiological data with regards to Asian women). Furthermore, women over the age of 35 years have an increased risk of pregnancy-related stroke.

Caesarean delivery has been shown to be associated with a 3-12 times increased risk of peripartum and postpartum stroke. ${ }^{411}$ This may be due, in part, to a higher likelihood of caesarean delivery among women who have had a stroke during pregnancy and other pregnancy-related conditions such as pre-eclampsia, which increases the risk of both stroke and caesarean section.

Known medical conditions strongly linked to stroke in pregnancy include hypertension, diabetes, sickle cell disease, thrombophilia, smoking and heart disease. Other risk factors in case series or reports are alcohol and recreational drug abuse, particularly cocaine. The presence of a lupus anticoagulant or anticardiolipin antibody is a well-recognised risk factor in pregnancy and an association with stroke in pregnancy has been shown with systemic lupus erythematosus. Other factors that may predispose to risk include multiple gestation and greater parity. At least one study has shown a greater association with migraine headaches; however, it is difficult to know whether this 
Table 1 Incidence of pregnancy-related strokes: comparison of published series

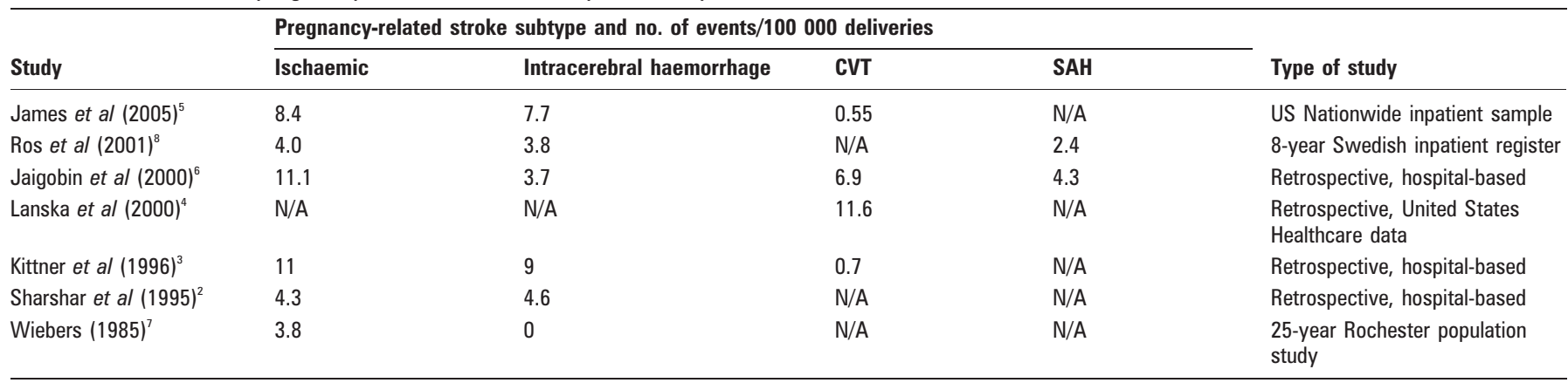

CVT, cerebral venous thrombosis; N/A, not applicable; SAH, subarachnoid haemorrhage.

relates simply to other associated disorders such as preeclampsia and eclampsia or is an independent risk factor in its own right.

Not surprisingly, complications of pregnancy such as preeclampsia, eclampsia, hyperemesis, and disturbances in electrolyte and fluid balance are significant risk factors for stroke in the context of pregnancy. In the study by Sharshar et al., ${ }^{2} 47 \%$ of non-haemorrhagic pregnancy-related strokes occurred in the context of toxaemia (six of fifteen ischaemic strokes had eclampsia and one had pre-eclampsia).

Patients undergoing blood transfusion have been shown to have a substantially higher incidence of stroke. This seems to relate to associated medical risk factors such as treatment for sickle cell disease and treatment for postpartum haemorrhage.

There have been a few case reports of arterial extra-cranial dissection occurring during or after pregnancy. In some cases, a strenuous labour has been the triggering event but this seems to be a relatively uncommon mechanism for stroke in this group. In some cases, dissection may be the consequence of a difficult and protracted delivery. ${ }^{12}{ }^{13}$ However, strenuous labour during delivery is not uncommon, so it seems likely that other factors are involved.

An antiphospholipid syndrome can be triggered by pregnancy. This may present with stroke, which may be either arterial or venous. Diagnosis rests on the detection of one or more antiphospholipid antibodies: lupus anticoagulant, anticardiolipin antibody and anti- $\beta 2$-glycoprotein. If the diagnosis is suspected, all three antibodies should be tested for. ${ }^{14}$ There is evidence in non-pregnant patients that anticoagulation is the treatment of choice in the presence of an antiphospholipid syndrome associated with arterial or venous thrombosis. ${ }^{15}{ }^{16}$ It would therefore seem advisable to treat pregnant patients in the same way. The treatment is predominantly anticoagulation with low-molecular-weight heparin (LMWH), transferring to oral anticoagulation when appropriate.

Women who are pregnant or in the postpartum period account for $10-25 \%$ of patients with thrombotic thrombocytopenic purpura (TTP). TTP may present with a wide variety of neurological presentations, including strokes, transient ischaemic attacks, fluctuating neurological symptoms, headaches, seizures and confusion. The diagnosis should be considered in pregnant women who develop anaemia and thrombocytopaenia. Blood film analysis may show red cell fragmentation and there may be a haemolytic pattern on laboratory analysis with elevated bilirubin, lactate dehydrogenase and increased reticulocyte count. Early diagnosis is critical as intensive treatment with plasma exchange may be life-saving. ${ }^{14}$
There are a few rare but specific complications of pregnancy that may cause stroke. ${ }^{17}$ The syndrome of amniotic fluid embolus is usually a catastrophic syndrome associated with sudden cardiovascular collapse, disseminated intravascular coagulation and neurological impairment. Strokes may occur secondary to venous or arterial events with, rarely, paradoxical amniotic emboli causing cardio-embolic strokes. ${ }^{18}$

Rarely, air embolus may result in ischaemic stroke as a complication of caesarean section or following intravaginal and intrauterine gas insufflation during pregnancy. ${ }^{19}$

Strenuous labour, multiparity, caesarean section, increased gestational age and increased maternal age all seem to be risk factors, although the syndrome may still occur in their absence. Most cases occur during labour and around the time of delivery.

Peripartum cardiomyopathy is a rare dilating cardiomyopathy than can present in the last month of pregnancy or within 5 months after delivery. ${ }^{20}$ It is more common in older, black or multiparous women. Up to $5 \%$ of such patients may develop stroke, usually secondary to cardiac thrombo-embolism, although watershed infarction may occur as a result of profound cardiac failure.

\section{PRE-ECLAMPSIA ${ }^{21}$ AND ECLAMPSIA}

The proportion of patients with pregnancy-associated stroke who have pre-eclampsia or eclampsia is between $25 \%$ and $45 \% .^{4511}$ The development of pre-eclampsia is characterised by hypertension, proteinuria and oedema. However, it is in fact a multisystem disorder that is characterised by widespread endothelial dysfunction and vasospasm. It is most likely to develop after the $20^{\text {th }}$ week of pregnancy but can also arise in the postpartum period. When seizures or coma develop in the context of pre-eclampsia, it is known as eclampsia. In addition to the neurological features of headaches, seizures and confusion, patients may also have sudden-onset focal neurological deficit consistent with stroke. Imaging may show arterial ischaemic events or intracerebral haemorrhage. Intracerebral haemorrhage seems to be a poor prognostic feature; this is probably due to the fact that its occurrence is associated with more significant pre-eclampsia (ie, higher blood pressure and greater endothelial damage).

The differential diagnosis includes cerebral venous thrombosis, which is more common in the postpartum period.

A reversible posterior leucoencephalopathy syndrome can occur as a consequence of eclampsia and pre-eclampsia. Clinically, there may be evidence of altered alertness and behaviour, seizures and visual loss, together with headaches and somnolence. On neuro-imaging, the most common abnormality 
is white matter oedema; this is seen as hyperintensity on MRI FLAIR images in the posterior cerebral hemispheres with sparing of the calcarine and paramedian occipital lobes. Posterior frontal cortical and subcortical white matter may also be involved, with involvement of the brainstem, cerebellum and basal ganglia being less common. One study suggested that partial or asymmetric MRI changes were more frequent in patients with eclampsia compared with other causes of posterior reversible leucoencephalopathy during pregnancy. ${ }^{22}$ Diffusion-weighted MRI is useful in patients with eclampsia to differentiate cytotoxic oedema occurring in the context of ischaemic infarction compared with reversible vasogenic oedema. ${ }^{23}$

It has been postulated that pre-eclampsia/eclampsia-associated stroke may be mediated by genetic factors that predispose to both endothelial dysfunction and to a thrombophilic state. Similarly, pre-eclampsia/eclampsia-associated haemorrhage may be associated with a disturbance of cerebral auto-regulation, which is partly genetically determined. A more straightforward link, of course, is hypertension, which predisposes to ischaemia and haemorrhage. The endothelial damage that is inherent in pre-eclampsia also increases the risk of thrombosis.

Treatment is aimed at lowering the blood pressure and rapid delivery of the baby. Seizures should be treated with magnesium sulphate and this may also be used prophylactically in preeclamptic patients. ${ }^{24-26}$

One recent study has shown an increased risk of subsequent non-pregnancy-related ischaemic stroke in women who develop pre-eclampsia. ${ }^{27}$ This would suggest that patients who develop pre-eclampsia should, subsequently, be closely monitored for stroke risk factors.

It is, of course, important to consider the numerous causes of stroke in the young, most of which have been reported in pregnancy. ${ }^{17}$ In many cases, it is likely that the occurrence during pregnancy is coincidental, although there are a few specific conditions that are associated with increased stroke risk in pregnancy. There is an increased risk of rheumatic heart disease recurrence in pregnancy, leading to a higher incidence of cardio-embolic disease. High-risk patients should be treated with antibiotic prophylaxis. It is also worth mentioning the relatively higher incidence of cardio-embolic events in pregnant patients with prosthetic heart valves-again, a result of the increased pro-thrombotic tendency of pregnancy. This can occur during conversion from warfarin to heparin and such patients require careful monitoring during pregnancy.

\section{PERIPARTUM OR POSTPARTUM ANGIOPATHY ${ }^{28-31}$}

This is a cerebral dysregulation syndrome affecting large- and medium-sized cerebral arteries. It may be associated with haemorrhagic or ischaemic stroke. The clinical picture typically occurs in women aged 20-50 years, and is usually one of abrupt onset with severe thunderclap headaches, seizures and focal neurological deficit. The imaging findings may often be suggestive as white matter changes have a posterior emphasis and are usually not as extensive compared with eclampsia. Diffusion-weighted MRI may also be helpful in differentiation. The same condition has also been called postpartum cerebral angiopathy when it develops in the puerperium. Migraine and the use of sympathomimetic or serotoninergic vasoactive drugs have been reported as risk factors in the development of peripartum and postpartum angiopathy. Although there is likely to be an overlap with eclampsia in terms of pathogenesis, patients do not have proteinuria and may not be hypertensive. It seems that cerebral vessel vasoconstriction is a relevant mechanism in some, but not all, cases.

\section{MANAGEMENT}

\section{Antiplatelet therapy}

Although some retrospective studies have concluded that aspirin use during the first trimester of pregnancy is associated with an increased risk of teratogenicity, this has not been confirmed in prospective studies. The potential risks of aspirin in later pregnancy include maternal and foetal haemorrhage, premature closure of the ductus arteriosus, and (theoretically) prolonged labour and delay in labour onset.

Low-dose aspirin $(75 \mathrm{mg}$ ) is not infrequently given throughout pregnancy in women who are at risk of pre-eclampsia. The safety of aspirin use during the first trimester remains contentious. In the CLASP study, ${ }^{32}$ aspirin was used from the second trimester without evidence of adverse effects in the mother or fetus, although there was a higher need for blood transfusion after delivery in the aspirin-treated group. However, it is, on balance, safe to use aspirin during pregnancy and whilst breastfeeding after an ischaemic stroke and should also be advised for any future pregnancy.

\section{Anticoagulant treatment}

In some circumstances, patients will require anticoagulation during pregnancy. The most common reasons for this include a known thrombophilic tendency or the presence of a prosthetic cardiac valve. The development of cerebral venous thrombosis during pregnancy and extracranial arterial artery dissection (which, as mentioned above, appears to be relatively uncommon in pregnancy) may also require anticoagulation. Heparin does not cross the placenta, whereas warfarin does. Heparin is therefore safe during pregnancy and whilst breastfeeding. It is important to monitor for the development of heparin-induced thrombocytopenia, which (despite the presence of heparin) results in a significant pro-thrombotic effect. This complication occurs in approximately $3 \%$ of patients receiving unfractionated heparin (UFH) and less in patients on fractionated treatment LMWH.

A large systematic review has shown that it is safe to use LMWH during pregnancy in patients with an underlying thrombophilic disorder. ${ }^{33}$ The optimal management of pregnant patients with prosthetic heart valves remains uncertain. Evidence-based guidelines ${ }^{34}$ have suggested three possible options: (1) either LMWH or UFH between 6 weeks and 12 weeks and close to term only, and to use warfarin at other times; (2) aggressive dose-adjusted UFH throughout pregnancy; or (3) aggressive adjusted-dose LMWH throughout pregnancy. If warfarin is used, the dose should be adjusted to attain a target International Normalized Ratio of 3.0 (range: 2.5 to 3.5).

LMWHs in prophylactic doses are not associated with an increased risk of severe bleeding peripartum, although higher doses (for example, in women with prosthetic heart valves) may increase the risk of maternal postpartum haemorrhage.

\section{Thrombolysis}

Pregnancy, and the 4 weeks after obstetric delivery, are generally regarded as exclusion criteria for thrombolysis following acute ischaemic stroke due to the risk of increased haemorrhage. There have, however, been a small number of case reports of intravenous and intra-arterial thrombolysis during pregnancy. ${ }^{35-39}$

In a number of cases, this has been carried out in the first trimester, often inadvertently.

A report of eight patients receiving thrombolysis during pregnancy ${ }^{38}$ comprised three patients receiving intravenous 
rt-PA, one receiving intra-arterial rt-PA, one receiving intraarterial urokinase and three receiving local urokinase (two of whom had a cerebral sinus thrombosis, both unusually in the first trimester). The mixed aetiologies and different forms of thrombolysis and administration make it difficult to draw any firm conclusions. Adverse outcomes in this study included one intrauterine haematoma; two asymptomatic, small, maternal intracranial haemorrhages; and one maternal death from massive cerebral infarction following arterial dissection complicating angioplasty. In the seven surviving mothers, three elected to have therapeutic abortions. There were two miscarriages occurring in the first trimester.

Intra-arterial thrombolysis using urokinase has been used in the immediate postpartum period in a patient who developed an acute middle cerebral artery thrombosis 15 hours after delivery. The patient made a rapid and uncomplicated recovery. ${ }^{39}$

In summary, the safety of thrombolysis in acute ischaemic stroke during pregnancy remains unproven. Notification of such patients in an international register may be a useful development to provide information on the expected neurological benefit against the possible risks of haemorrhage in pregnancy.

\section{Cerebral venous sinus thrombosis}

Pregnancy and, in particular, the puerperium carry an increased risk of cerebral venous thrombosis (CVT), with a fatality rate ranging from 4 to $36 \%{ }^{40} 41$ In the majority of patients, symptoms develop within 3 weeks after delivery. Approximately $2 \%$ of strokes occurring during pregnancy can be attributed to venous thrombosis. The incidence is higher in under-developed countries. Dehydration is an important and preventable additional risk factor, in addition to the increased risk that is inherent in being pregnant. There is also a higher incidence of anaemia in patients with CVT related to pregnancy than in non-obstetric-related cases. Cases can occur during pregnancy, although are much less common. Typical presenting features include headache, disturbance of consciousness, focal neurological signs and seizures. Papilloedema has been reported in only half of cases and, in this context, is not a particularly reliable sign of raised intracranial pressure. The involvement of cortical veins may lead to one or more areas of venous infarction with or without haemorrhagic transformation. The presentation in such cases is often with localisation-related seizures and focal neurological deficits, depending on the territories involved. Deterioration in conscious level suggests either multiple lesions in the cerebral hemispheres, bilateral thalami or, more worryingly, transtentorial herniation and brain-stem compression. A stroke-like presentation has been described as a manifestation of cortical-vein thrombosis. ${ }^{42}$

In a retrospective review of 67 cases of CVT occurring predominantly in the puerperium, ${ }^{40}$ the authors noted that the patients tended to be younger (mean age of 26 years compared with age 36 years). There seemed to be a tendency to a better outcome in the obstetric group with a lower mortality. Despite the strong association with the postpartum state, it is still advisable that such patients have a full thrombophilia screen to exclude any additional pro-thrombotic tendency. One report showed a link between puerperal CVT and protein S deficiency. $^{43}$

Neuro-radiological features include venous infarction with or without haemorrhage.

CT imaging of the brain may show venous infarcts with or without haemorrhage, which do not conform to an arterial distribution. Hyperdensity in the sagital sinus on an unenhanced
CT scan due to occlusion from fresh thrombus - the so-called delta sign-may be a useful clue. MR studies may be useful in identifying CVT, either with conventional studies with or without additional MR venography. The conventional treatment for CVT is anticoagulation, although firm evidence of its benefits is lacking. A number of studies during pregnancy have suggested improvement in survival following anticoagulation, ${ }^{44}{ }^{45}$ although the numbers studied have been small and did not achieve statistical significance.

One randomised study ${ }^{46}$ has compared the effect of intravenous UFH versus placebo in a cohort of 57 women from India with sinus thrombosis in the puerperium. Diagnosis was not confirmed with MRI or angiography, and again showed a nonsignificant benefit of anticoagulant treatment compared with placebo.

Concomitant intracranial haemorrhage related to CVT (which is present in $40 \%$ of patients even before treatment) is not a contraindication for heparin therapy. ${ }^{47-49}$ The optimal duration of oral anticoagulation after the acute phase is unclear. Oral anticoagulation may be given for 3 months if CVT is secondary to a transient risk factor such as the puerperium.

In general, full heparinisation is safe once 24 hours have elapsed since delivery, and warfarin therapy may be started 2-3 days after birth. Both heparin and warfarin are safe whilst breastfeeding.

Thrombophilia screening should be carried out 1 month after stopping anticoagulant treatment and no earlier than 6 weeks post-natally (because of the effects on factors such as protein S, which may be temporarily low during pregnancy and for 6 weeks after birth). A study of 16 consecutive patients with CVT included three patients who were postpartum and one who was pregnant at the time of the event. All had an additional identifiable prothrombotic tendency, such as Protein $\mathrm{S}$ or $\mathrm{C}$ deficiency and Factor $\mathrm{V}$ leiden mutation. ${ }^{50}$

\section{Haemorrhage}

Intracranial haemorrhage has been estimated to constitute $2-7 \%$ of the total cases of neurological disorders related to pregnancy. In many cases, this relates to uncontrolled hypertension.

Of the 34 cases of stroke related to pregnancy described by Jaigobin et al., 613 were haemorrhages, seven of which were subarachnoid haemorrhage (SAH). Three of these patients had rupture of an underlying cerebral aneurysm, all of which were posterior communicating artery aneurysms. Haemorrhage from either a subarachnoid bleed or intracerebral bleed occurred most commonly in the second trimester and the postpartum period. A bleed from an underlying aneurysm occurred with equal frequency in all trimesters and the puerperium, whereas bleeding from an underlying arteriovenous malformation (AVM) occurred after the first trimester and in the postpartum period.

$\mathrm{SAH}$, mostly from aneurysms, accounts for $3 \%$ of all strokes. A saccular aneurysm will be responsible in approximately $85 \%$ of patients with SAH. In $10 \%$ of patients, SAH is secondary to non-aneurysmal perimesencephalic haemorrhage, which should be considered when haemorrhage on imaging is predominantly confined to the basal cisterns around the midbrain. Other rarer causes include septic aneurysm, arterial dissection, cerebral AVM, dural arteriovenous fistula and vascular lesions around the spinal cord. Pituitary apoplexy should also be considered, particularly if physical examination reveals a field defect, reduction of visual acuity or complex ophthalmoplegia. 
Haemorrhagic infarction of a previously asymptomatic pituitary tumour may be precipitated by pregnancy.

A rare cause of intracerebral haemorrhage to consider in pregnancy is choriocarcinoma, a malignant tumour of gestational trophoblasts that most commonly metastasise to the lungs and liver but also to the brain. The metastases are frequently haemorrhagic and so may present during pregnancy with an intracerebral or SAH. ${ }^{51}$ Furthermore, trophoblasts from metastatic brain lesions may invade cerebral vessels, leading to cerebral infarction. Choriocarcinoma normally occurs in the context of a molar pregnancy, but may also follow an apparently normal delivery, abortion or ectopic pregnancy.

\section{AVM}

In women with a previously asymptomatic AVM, the risk of a haemorrhage occurring during pregnancy is approximately $3.5 \%{ }^{52}$ Pregnancy does not seem to increase the risk of first cerebral haemorrhage from an AVM. For this reason, it would seem reasonable to defer treatment of an underling AVM that has not bled until after delivery. With regards methods of delivery in such patients, it makes sense to try and avoid a strenuous and painful labour, both of which could lead to large surges in blood pressure and possible haemorrhage. We would therefore advocate either elective caesarean section or vaginal delivery with the use of epidural anaesthesia to reduce pain, together with instrumentation (if necessary) to reduce pushing.

In those patients who have presented with a bleed during pregnancy, the decision for interventional treatment is more difficult. An older retrospective study showed no improvement in maternal or foetal outcome after surgery. ${ }^{53}$ However, the current treatment for AVMs is primarily endovascular intervention, which has a relatively low risk of mortality and morbidity.

Some patients may have an AVM that is not surgically or endovascularly remediable. The mode of delivery in such patients is controversial. In the retrospective analysis of the literature, ${ }^{53}$ for those patients with an AVM not operated on, there was no difference in maternal or foetal outcome between caesarean or vaginal delivery. Some case reports have advocated caesarean section to avoid the risks of hypertension related to the valsalva manoeuvre in vaginal delivery. However, the risks of anaesthesia also have to be considered. On balance, we would advocate elective caesarean section. If a vaginal delivery is undertaken, we would recommend epidural anaesthesia and instrumentation to shorten delivery.

A patient with a treated AVM in which the feeding vessels have all been occluded can undergo a normal labour and delivery.

\section{CEREBRAL ANEURYSM}

The incidence of cerebral aneurysms in the general population is relatively high-between 3.6 and $6 \% .{ }^{54}$ Approximately 1 in 10000 pregnancies is complicated by the rupture of an intracranial aneurysm. This is most likely to occur in the second or third trimester. Although there are no formal studies, it seems unlikely that pregnancy itself greatly alters the natural history of a previously asymptomatic cerebral aneurysm, given the relative infrequency of rupture during pregnancy. It would therefore seem reasonable to defer treatment (either with surgery or, now more commonly, with endovascular intervention) in asymptomatic patients until after delivery. Elective surgical clipping of asymptomatic aneurysms has a morbidity of $10.9 \%$ and a mortality of $3.8 \%$. Treatment of aneurysms by coiling, for which there is less long-term follow-up data available, has a $4 \%$ morbidity and a $1 \%$ mortality, but only achieves complete aneurysm occlusion in $52-78 \%$ of cases. ${ }^{54}$

In patients who have bled from an aneurysm during pregnancy, treatment with endovascular coiling is now the preferred treatment of choice, ${ }^{556}$ and such patients should be treated prior to delivery.

The method of delivery in patients with a cerebral aneurysm that has not bled should be based on obstetric considerations as there is no evidence that maternal or foetal outcome is improved by caesarean section compared with closely supervised vaginal delivery. In the absence of any convincing data to support one method of delivery over another, the authors tend to favour elective caesarean section. However, instrumental delivery (with forceps or ventouse) under epidural anaesthesia can also be used to limit pushing in the second stage of labour.

Although epidural anaesthesia is generally regarded as safe, there have been a few reports of intracranial or SAH occurring following dural puncture. Presumably, prolonged low cerebrospinal fluid pressure can lead to an increase in transmural pressure across an arterial wall, facilitating rupture of an aneurysm or AVM. For this reason, insertion of an epidural catheter in this context should be inserted by an experienced operator to reduce the risk of a dural tap.

\section{RISK OF STROKE RECURRENCE}

The overall risk of recurrence of stroke associated with subsequent pregnancies is relatively small. In a multi-centre study of 489 women presenting with cerebrovascular events between the ages of 15 and 40 years, 28 (of 373 patients) had the initial ischaemic event during pregnancy or during the puerperium. Thirteen of the whole cohort had a recurrent stroke but only two of these occurred in a subsequent pregnancy. There were no instances of recurrence in those patients presenting with cerebral venous thrombosis, although one patient in this group had a subsequent arterial stroke that was unrelated to pregnancy. However, these patients have an approximate risk of recurrent stroke of $1 \%$ in the following 12 months and $2.3 \%$ within 5 years, ${ }^{57}$ so assessment for treatable risk factors (including future pregnancy) would seem prudent. Thromboprophylaxis in any future pregnancy (and the puerperium) is advisable.

\section{CONCLUSION}

Although uncommon, the development of stroke during pregnancy and the puerperium can be a challenging diagnostic and management problem. For this reason, we would reiterate that such patients are managed in specialised multi-disciplinary units with rapid access to expertise in obstetrics, neurology, neuro-radiology and rehabilitation services.

Competing interests: None declared.

\section{REFERENCES}

1. Department of Health, Scottish Executive Health Department, and Department of Health, Social Services and Public Safety, Northern Ireland. Why Mothers Die. Sixth Report of Confidential Enquiries into Maternal Deaths in the United Kingdom, 2000-2002.London: RCOG Press, 2004.

2. Sharshar T, Lamy C, Mas J. Incidence and causes of strokes associated with pregnancy and puerperium. A study in public hospitals of lle de France. Stroke 1995:26:930-6.

3. Kittner SJ, Stern BJ, Feeser BR, et al. Pregnancy and the risk of stroke. N Engl J Med 1996;335:768-74.

4. Lanska DJ, Kryscio RJ. Risk factors for peripartum and postpartum stroke and intracranial venous thrombosis. Stroke 2000;31:1274-82.

5. James $\mathbf{A H}$, Bushnell CD, Jamison MG, et al. Incidence and risk factors for stroke in pregnancy and the puerperium. Obstet Gynecol 2005;106:509-16. 
6. Jaigobin C, Silver FL. Stroke and pregnancy. Stroke 2000;31:2948-51.

7. Wiebers DO, Whisnant JP. The incidence of stroke among pregnant women in Rochester Minn, 1955 through 1979. JAMA 1985;254:3055-7.

8. Ros HS, Lichtenstein P, Bellocco R, et al. Increased risks of circulatory diseases in late pregnancy and puerperium. Epidemiology 2001;12:456-60.

9. Pettiti DB, Sidney S, Quesenberry CP, et al. Incidence of stroke and myocardial infarction in women of reproductive age. Stroke 1997;28:280-3.

10. Chang J, Elam-Evans LD, Berg CJ, et al. Pregnancy-related mortality surveillanceUnited States, 1991-1999. MMWR Surveill Summ 2003:52:1-8.

11. Ros HS, Lichtenstein P, Bellocco R, et al. Pulmonary embolism and stroke in relation to pregnancy: How can high-risk women be identified? Am J Obstet Gynecol 2002;186:198-203.

12. Wiebers D0, Mokri B. Internal carotid artery dissection after childbirth. Stroke 1985;16:956-9.

13. Oehler J, Lichy C, Gandjour J, et al. Dissection of four cerebral arteries after protracted birth. Nervenarzt 2003;74:366-9.

14. Austin S, Cohen H, Losseff L. Haematology and neurology. J Neurol Neurosurg Psychiatry 2007:78:334-41.

15. Rosove MH, Brewer PMC. Antiphospholipid thrombosis: clinical course after the first thrombotic event in 70 patients. Ann Intern Med 1992;117:303-8.

16. Khamashta MA, Cuadrado MJ, Mujic F, et al. The management of thrombosis in the antiphospholipid antibody syndrome. N Engl J Med 1995;332:993-7.

17. Mas JL, Lamy C. Stroke in pregnancy and the puerperium. J Neurol 1998;245:1431-59.

18. Moore J, Baldisseri MR. Amniotic fluid embolus. Critical Care Medicine. Crit Illness Preg 2005:33:S279-S285

19. Muth CM, Shank ES. Gas embolism. N Engl J Med 2000;342:476-82

20. Pearson GD, Veille JC, Rahimtoola S, et al. Peripartum cardiomyopathy: National Heart, Lung, and Blood Institute and Office of Rare Diseases (National Institutes of Health) workshop recommendations and review. JAMA 2000;283:1183-8.

21. Sibai B, Dekker G, Kupferminc M. Pre-eclampsia. Lancet 2005;365:785-99.

22. Bartynski WS, Boardman JF. Distinct imaging patterns and lesion distribution in posterior reversible encephalopathy syndrome. Am J Neuroradiol 2007;28:1320-7.

23. Schaefer PW, Buonanno FS, Gonzalez G, et al. Diffusion-weighted imaging discriminates between cytotoxic oedema and vasogenic oedema in a patient with eclampsia. Stroke 1997;28:1082-5

24. Sawle GV, Ramsay MM. The neurology of pregnancy. J Neurol Neurosurg Psychiatr 1998;64:717-25

25. Anon. Which anticonvulsant for women with eclampsia? Evidence from the Collaborative Eclampsia Trial. Lancet 1995:345:1455-63.

26. Altman D, Carroli G, Duley L, et al. Do women with pre-eclampsia, and their babies, benefit from magnesium sulphate? The Magpie Trial: a randomised placebo-controlled trial. Lancet 2002;359:1877-90.

27. Brown DW, Dueker ND, Jamieson DJ, et al. Pre-eclampsia and the risk of ischemic stroke among young women: results from the stroke prevention in young women study. Stroke 2006;37:1055-9.

28. Call GK, Fleming MC, Sealfon S, et al. Reversible cerebral segmental vasoconstriction. Stroke 1988;19:1159-70.

29. Raps EC, Galetta SL, Broderick M, et al. Delayed peripartum vasculopathy: cerebral eclampsia revisited. Ann Neurol 1993;33:222-5.

30. Donaldson J0. Eclampsia and postpartum cerebral angiopathy [editorial]. J Neurol Sci 2000;178:1.

31. Singhal AB. Postpartum angiopathy with reversible posterior leukoencephalopathy. Arch Neurol 2004;61:411-6.

32. Anon. CLASP: a randomised trial of low-dose aspirin for the prevention and treatment of pre-eclampsia among 9364 pregnant women. CLASP (Collaborative Low-dose Aspirin Study in Pregnancy) Collaborative Group. Lancet 1994;343:619-29.

33. Greer IA, Nelson-Piercy C. Low-molecular-weight heparins for thromboprophylaxis and treatment of venous thromboembolism in pregnancy: a systematic review of safety and efficacy. Blood 2005;106:401-7.
34. Bates SM, Greer IA, Hirsh J, et al. Use of antithrombotic agents during pregnancy The seventh ACCP conference on antithrombotic and thrombolytic therapy. Chest 2004; 126:627S-644S

35. Elford K, Leader A, Wee R, et al. Stroke in ovarian hyperstimulation syndrome in early pregnancy treated with intra-arterial rt-PA. Neurology 2002;59:1270-2.

36. Dapprich $\mathbf{M}$, Boessenecker W. Fibrinolysis with alteplase in a pregnant woman with stroke. Cerebrovasc Dis 2002;13:290.

37. Johnson DM, Kramer DC, Cohen E, et al. Thrombolytic therapy for acute stroke in late pregnancy with intra-arterial recombinant tissue plasminogen activator. Stroke 2005;36:e53-e55

38. Murugappan A, Coplin WM, Al-Sadat AN, et al. Thrombolytic therapy of acute ischemic stroke during pregnancy. Neurology 2006;66:768-70.

39. Méndez JC, Masjuán J, García N,et al. Successful intra-arterial thrombolysis for acute ischemic stroke in the immediate postpartum period: Case Report. Cardiovasc Intervent Radiol 2006 Sep 19; [Epub ahead of print].

40. Cantu C, Barinagarrementeria F. Cerebral venous thrombosis associated with pregnancy and puerperium. Review of 67 cases. Stroke 1993;24:1880-4.

41. Canhao P, Ferro J, Lindgren A, et al. Causes and predictors of death in cerebral venous thrombosis. Stroke 2005;36:1720-5.

42. Jacobs K, Moulin T, Bogousslavsky J, et al. The stroke syndrome of cortical vein thrombosis. Neurology 1996;47:376-82.

43. Galan HL, McDowell AB, Johnson PR, et al. Puerperal cerebral venous thrombosis associated with decreased free protein S: a case report. J Reprod Med 1995; 40:859-62.

44. Einhaupl KM, Villringer $A$, Meister $\mathbf{W}$, et al. Heparin treatment in sinus venous thrombosis. Lancet 1991:338:597-600.

45. De Bruijn SF, Stam J. Randomized, placebo-controlled trial of anticoagulant treatment with low-molecular weight heparin for cerebral sinus thrombosis. Stroke 1999;30:484-8

46. Nagaraja D, Rao BSS, Taly AB, et al. Randomized controlled trial of heparin in puerperal cerebral venous/sinus thrombosis. Nimhans J 1995;13:111-5.

47. Brucker MB, Vollert-Rogenhofer $\mathrm{H}$, Wagner M., et al. Heparin treatment in acute cerebral sinus venous thrombosis: a retrospective clinical and MR analysis of 42 cases. Cerebrovasc Dis 1998;8:331-3.

48. Fink JN, McAuley DL. Safety of anticoagulation for cerebral venous thrombosis associated with intracerebral hematoma. Neurology 2001;57:1138-9.

49. Einhaupl K, Bousser MG, de Bruijn SF, et al. EFNS guideline on the treatment of cerebral venous and sinus thrombosis. Eur J Neurol 2006;13:553-9.

50. Cakmak S, Derex L, Berruyer M., et al. Cerebral venous thrombosis. Clinical outcome and systematic screening of prothrombotic factors. Neurology 2003:60:1175-8.

51. Huang CY, Chen $\mathrm{CA}$, Hsieh $\mathrm{CY}$, et al. Intracerebral hemorrhage as initial presentation of gestational choriocarcinoma: a case report and literature review. Int J Gynecol Cancer 2007;17:1166-71.

52. Horton JC, Chambers WA, Lyons SL, et al. Pregnancy and the risk of hemorrhage from cerebral arteriovenous malformations. Neurosurgery 1990;27:867-71.

53. Dias MS, Sekhar LN. Intracranial haemorrhage from aneurysms and arteriovenous malformations during pregnancy and the puerperium. Neurosurgery 1990;27:855-66.

54. Wardlaw JM, White PM. The detection and management of unruptured intracrania aneurysms. Brain 2000;123:205-21.

55. Meyers PM, Halbach VV, Malek AM, et al. Endovascular treatment of cerebral artery aneurysms during pregnancy: report of three cases. Am J Neuroradiol 2000;21:1306-11.

56. Piotin M, Filho CB, Rothimbakam R, et al. Endovascular treatment of acutely ruptured intracranial aneurysms in pregnancy. Case Reports. Am J Obstet Gynecol 2001; 185:1261-2.

57. Lamy C, Hamon JB, Coste J, et al. Ischemic stroke in young women. Risk of recurrence during subsequent pregnancies. Neurology 2000;55:269-74. 